\title{
Ribozymes and RNA Catalysis, edited by David M. Lilly and Fritz Eckstein. 2007. RSC Publishing, Cambridge, UK. ISBN (online): 978-1-84755-798-8, ISBN (print): 978-0-85404-253-1.
}

I recall as a graduate student being excited reading the first papers describing the discovery of RNA catalysis as well as the commentary on the enzymological and evolutional implications that followed. I feel a bit sorry for students in my laboratory who share a fascination with ribozymes, but for whom RNA catalysis is a fact learned in undergraduate biochemistry class. This book, however, through a series of expert reviews, explores the forefront of ribozyme research while evoking the excitement of those early discoveries. One reason for this attribute is that this is an update of a volume edited by Drs. Eckstein and Lilley 10 years ago, and their scholarly perspective on how the field has grown and developed is reflected in the choice of themes and the spirit of the volume. The foremost example is the introduction by Tom Cech, who received the Nobel Prize along with Sid Altman for the discovery of RNA catalysts, in which he contrasts predictions made on the structure and mechanism of ribozymes at the time of their discovery to the "modern" perspective.

The first third of the volume discusses small self-cleaving ribozyme structure and function with appropriate emphasis on the importance of acid-base catalysis. A defining characteristic of ribozyme enzymology that is reflected in most of the reviews, but especially so in those on small ribozymes, is the facility for probing individual functional groups by site-specific modification. The well-developed field of nucleotide chemistry combined with the facility of solid phase synthesis permits structure-function studies to be done at essentially atomic resolution. One of the most exciting areas of development is exploring how RNAs facilitate proton transfer resulting in transition state stabilization. Detailed mechanistic aspects of proton transfer can be difficult to digest, but Phil Bevilacqua presents a clear and concise overview of proton transfer chemistry (scandalously invoking William Jencks' "libido" rule in the process), the driving forces for $p K_{a}$ shifting, and considerations for the quantitative contribution of proton transfer to rate enhancement. This information serves as a context

Article published online ahead of print. Article and publication date are at http://www.rnajournal.org/cgi/doi/10.1261/rna.1047008. for the case by case studies of individual small ribozymes that are explored in more detail in subsequent chapters.

Like all fields of science there are a few great "stories" in which competing or exclusive theories arise that are later insightfully resolved by elegant experimental solutions that in hindsight seem obvious. The evolution of understanding the structure and function of the hammerhead ribozyme is such a narrative, and Bill Scott gives the insider's view of how it unfolded (or folded, eventually). Dominic Lambert and John Burke extend this theme by focusing in greater detail on analysis and modeling of the hammerhead ribozyme active site. Subsequent chapters by Joe Piccirilli and colleagues and by D.M.J. Lilley integrate the structural and functional information leading to the current mechanistic framework for the HDV, hairpin, and VS ribozymes. Highlighted for veterans of ribozyme research and newbies alike in these chapters is the difficulty in pinning down the specific role of a functional group as an acid or base catalyst. The high degree of linkage between conformational dynamics and observed rates of catalysis is also tied in well. Together with related chapters on relative newcomers, the glmS riboswitch riboyme by Link and Breaker and mammalian self-cleaving ribozymes by Andrej Luptak and Jack Szostak, the essential features of small ribozyme research relating to catalysis are well covered.

A second major section is devoted to chapters exploring the large ribozymes, RNase P RNA and Group I and Group II introns. Here, the coordination of new structural perspectives with incisive biochemical analyses to understand the mechanism takes center stage with a particular focus on the role of active site metal ions. For the bacterial RNase $\mathrm{P}$ ribozyme the big story is the elucidation of threedimensional structures of its RNA and protein subunits. Norm Pace, himself a direct participant in the birth of ribozymology, and his colleagues review substrate positioning and active site-probing data in the context of this new structural perspective (with a characteristically healthy dose of phyolgenetic comparative analyses). For the Group I intron ribozymes Barb Golden provides a balanced and entertaining review of the integration of high-resolution structures in the development of models for the active site 
of this ribozyme. Essential material relating the coordination of biochemical studies, such as thiophilic metal rescue analyses, with active site metal ion interactions in highresolution structures of the active site is well covered here. This serves as the basis for interesting explorations of what constitutes a catalytic site in RNA and how complex RNA catalytic mechanisms may have evolved. Group II introns are given a comprehensive treatment by Anna Pyle from their complex secondary and tertiary structures revealed by insightful biochemical and comparative sequence analyses to current models for active site architecture and the role of protein maturases. Among the gems found throughout these chapters is the inclusion of a not entirely tonguein-cheek argument for GII introns as "tiny living things." It makes me wonder whether the slow pace of research in my laboratory could be due to the wicked designs of these creatures after all.

A strength of the volume, which makes it both interesting to the expert and highly useful for the newcomer, is the inclusion of chapters on the spliceosome and ribosome. The spliceosome has yet to receive widespread recognition as a ribozyme (indeed, the title of the chapter by Dipali Sashital and Sam Butcher is punctuated by a question mark). However, many of the fundamental properties of ribozymes have proven useful in understanding spliceosomal RNA function and dynamics and defining the features that a spliceosomal active site must possess-features that are expertly discussed in terms of emerging high-resolution structures of portions of snRNAs. Ribosome enzymology is another emergent field, and the chapter by Marina Rodnina adroitly outlines how the mechanism of the peptidyl transferase center is defined by the fundamental chemistry of aminolysis. Indeed, this chapter is exemplary from the standpoint of building up an understanding of mechanisms from first (chemical) principles.

Coverage of ribozyme structure and mechanism would not be complete without consideration of RNA folding, given the obvious linkage between structure and function and the ability of RNA to assume multiple stable conformations. Others have pointed to the fact that this may be a property that is useful for RNA function in multistep reactions and in achieving specificity. RNA folding is, in its own right, a subject worthy of an entire volume, but again, for the student or chemist/biologist entering the field, the main issues are defined and expertly explored by Sarah Woodson and Prashanth Rangan. A strength of their chapter is that the authors comprehensively cover the most useful biophysical and molecular approaches, making it an ideal roadmap for students and researchers who are dissecting conformational changes in other systems.

Inevitably, there are areas for which a biased reader (such as me) would desire greater coverage. Given the complexity of the issues involved, one theme that could be developed further in an integrative chapter like the one by Bevilacqua is the interaction of RNA and metal ions. Nonetheless, chapters by Golden, Pace, Pyle and others cover the important issues and cite comprehensive reviews that are excellent. Also, further information on the chemistry of phosphoryl transfer would be useful since diagrams of ribozyme chemical mechanism included throughout the volume show some interesting variety in the bond order, charge, and protonation states. In sum, this is a great resource for advanced students and researchers getting a grip on the major issues and advances in the field. Indeed, an excellent and concise primer to the key issues and themes addressed by the volume is included as Chapter 1 . It is also valuable as a source of comprehensive reviews of the major experimental systems to use for reference and as an entry into the literature for these systems. For the new students in our laboratory, it is now required reading.

Mike Harris, Center for RNA Molecular Biology, Case Western Reserve University School of Medicine, Cleveland, $\mathrm{OH}$ 44106, USA; e-mail: meh@cwru.edu; fax: (216) 368-2010. 

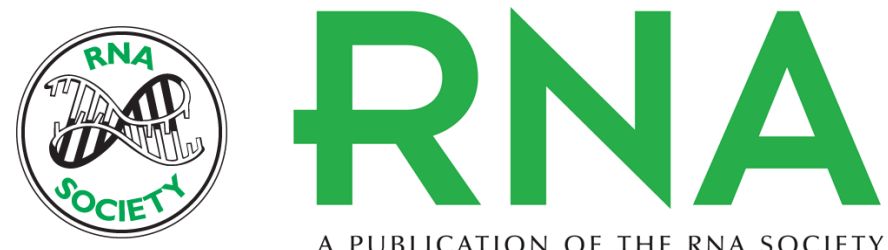

A PUBLICATION OF THE RNA SOCIETY

\section{Ribozymes and RNA Catalysis, edited by David M. Lilly and Fritz Eckstein. 2007. RSC Publishing, Cambridge, UK. ISBN (online): 978-1-84755-798-8, ISBN (print): 978-0-85404-253-1.}

Mike Harris

RNA 2008 14: 1003-1004

Supplemental http://rnajournal.cshlp.org/content/suppl/2008/05/23/rna.1047008.DC1

Material

License

Email Alerting Receive free email alerts when new articles cite this article - sign up in the box at the Service top right corner of the article or click here. 